\title{
On the Complexity of Nash Equilibria of Action-Graph Games
}

\author{
Constantinos Daskalakis ${ }^{\star}$, Grant Schoenebeck ${ }^{\star \star}$, Gregory Valiant ${ }^{\star \star \star}$, and Paul Valiant ${ }^{\dagger}$
}

\begin{abstract}
We consider the problem of computing Nash Equilibria of action-graph games (AGGs). AGGs, introduced by Bhat and Leyton-Brown, is a succinct representation of games that encapsulates both 'local'dependencies as in graphical games, and partial indifference to other agents' identities as in anonymous games, which occur in many natural settings. This is achieved by specifying a graph on the set of actions, so that the payoff of an agent for selecting a strategy depends only on the number of agents playing each of the neighboring strategies in the action graph. We present a Polynomial Time Approximation Scheme for computing mixed Nash equilibria of AGGs with constant treewidth and a constant number of agent types (and an arbitrary number of strategies), together with hardness results for the cases when either the treewidth or the number of agent types is unconstrained. In particular, we show that even if the action graph is a tree, but the number of agent-types is unconstrained, it is NP-complete to decide the existence of a pure-strategy Nash equilibrium and PPAD-complete to compute a mixed Nash equilibrium (even an approximate one); similarly for symmetric AGGs (all agents belong to a single type), if we allow arbitrary treewidth. These hardness results suggest that, in some sense, our PTAS is as strong of a positive result as one can expect.
\end{abstract}

\section{Introduction}

What is the likely behavior of autonomous agents in a variety of competitive environments? This question has been the motivation for much of economic theory. Partly due to the increasing prevalence of vast online networks over which millions of individuals exchange information, goods, and services, and the corresponding increasing importance of understanding the dynamics of such interactions, the Computer Science community has joined in the effort of studying game-theoretic questions.

Computing equilibria in games and markets has been extensively studied in the Economics and Operations Research communities since the 1960 's, see e.g. [17,21, 26, 22, 24]. Computational tractability has been recently recognized as an important prerequisite for modeling competitive environments and measuring the plausibility of solution concepts in Economics: if finding an equilibrium is computationally intractable, should we believe that it naturally arises? And, is it plausible that markets converge to solutions of computationally intractable problems? Probably not — but if so, we should certainly know about it.

Computing Nash equilibria in games, even in the case of two players, has been recently shown to be an intractable problem; in particular, it was shown to be complete for the class of fixed point computation problems termed PPAD [8, 3]. This result on the intractability of computing Nash equilibria has sparked considerable effort to find efficient algorithms for approximating such equilibria, and has increased the importance of considering special classes of games for which Nash equilibria might be efficiently computable.

For two-player games the hardness of computing approximate equilibria persists even if the required approximation is inverse polynomial in the number of strategies of the game [4]; similarly, hardness persists in graphical games if the required approximation is inverse polynomial in the number of players $[8,4]$. The same hardness results apply to special cases of the problem, e.g. win-lose games, where the payoff values of the game are restricted to $\{0,1\}$ [6], sparse bimatrix games, where the number of non-zero entries of each row an column of the payoff matrices is a constant, and two-player symmetric games [14]. The emerging question of the research in this field is: Is there a Polynomial Time Approximation Scheme (PTAS) for Computing Nash Equilibria? And, which special cases of the problem are computationally tractable?

The zero-sum two-player case is well-known to be tractable by reduction to linear programming [25]. Tractability persists in the case of low-rank two-player games, in which the sum $A+B$ of the payoff matrices of the players, instead of being 0, has fixed rank; in this case, a PTAS exists for finding mixed Nash equilibria [16]. In $n$-player graphical

\footnotetext{
* University of California, Berkeley. Email: costisecs . berkeley.edu.

${ }^{\star \star}$ University of California, Berkeley. Email: grant@eecs . berkeley . edu.

${ }^{\star \star \star}$ University of California, Berkeley. Email: gvaliant @eecs . berkeley . edu.

${ }^{\dagger}$ MIT. Email: pvaliantemit.edu.
} 
games, a PTAS has been provided if the tree-width is $O(\log n)$ and the maximum degree is bounded [9]; in the case of dense graphical games, a quasi-polynomial time approximation scheme exists [11].

An important line of research on tractable special cases has explored games with symmetries. Mutli-player symmetric games with about logarithmically few strategies per player can be solved exactly in polynomial time by reduction to the existential theory of reals [20]. For congestion games, a pure Nash equilibrium can be computed in polynomial time if the game is a symmetric network congestion game [13], and an approximate pure Nash equilibrium if the game is symmetric but not necessarily a network game and the utilities satisfy a "bounded-jump condition" [5]. Another important class of games for which computing an approximate equilibrium is tractable is the class of anonymous games, in which each player is different, but does not care about the identities of the other players, as it happens for example congestion games, certain auction settings, and social phenomena [2]; a PTAS for anonymous games with a fixed number of strategies has been provided in $[10,11]$. For a thorough study of the problem of computing pure Nash equilibria in symmetric and anonymous games see [12].

In this paper, we consider another special class of games, Action Graph Games (AGGs), that were introduced by Bhot and Leyton-Brown [1]. AGGs is a fully general game representation that succinctly captures both 'local' dependencies as in graphical games, as well as partial indifference to other agents' identities as in anonymous games. Strategies are represented as nodes in a graph, called strategy graph, and the utility of an agent for selecting a strategynode depends on the number of other agents selecting each of the neighboring strategies. The only attribute that distinguishes players is the set of strategies that each player is allowed to play. In particular, all agents who play a given strategy get the same payoff. A variety of natural games can be concisely represented as AGGs, and we refer the reader to $[1,15]$ for further discussion.

In the remainder of this section, we discuss previous work on AGGs and summarize our results. In the end of the section, we provide definitions.

\subsection{Previous Work}

Action graph games were first defined by Bhat and Leyton-Brown [1] who considered the problem of computing Nash equilibria of these games. In particular, they analyzed the complexity of computing the Jacobian of the payoff function-a computation that is, in practice, the bottleneck of the continuation method of computing a Nash equilibrium. They considered this computation for both general AGGs and AGGs with a single player type (symmetric AGGs), and found that this computation is efficient in the latter case. For pure Nash equilibria, Jiang and LeytonBrown [15] show that deciding the existence of such equilibria in AGGs is NP-complete, even in the case of a single player type and bounded degree. On the other hand, they provide a polynomial time algorithm for finding pure-Nash equilibria in AGGs with constant number of player types when the strategy graph has bounded tree-width.

\subsection{Our Results}

We examine, and largely resolve the computational complexity of computing Nash equilibria in action graph games. We give a polynomial algorithm for computing an $\epsilon$-Nash equilibrium for AGGs with constant treewidth and degree and a constant number of agent types (and arbitrarily many strategies), together with hardness results for the cases when either the treewidth or the number of agent types is unconstrained. In particular, we show that even if the strategy graph is a tree with bounded degree but the number of agent types is unconstrained, it is NP-complete to decide the existence of a pure-strategy Nash equilibrium and PPAD-complete to compute a mixed Nash equilibrium; similarly for AGGs in which all agents are a single type if we allow the strategy graph to have arbitrary treewidth. These hardness results suggest that, in some sense, our PTAS is as strong of a positive result as one can expect. While Bhat and Leyton-Brown studied heuristics for computing mixed Nash equilibria [1], the authors know of no previous complexity theoretic results concerning computing mixed Nash equilibria for AGGs apart from the PPAD-hardness result that follows from them being a generalization of normal-form games.

\subsection{Definitions}

In this section we give a formal definition of AGGs and introduce the terminology that will be used in the remainder of this paper. We follow the notation and terminology introduced in [15]. 
Definition 1. An action-graph game, $A$, is a tuple $\langle P, \mathbf{S}, G, u\rangle$ where

- $P:=\{1, \ldots, n\}$ is the set of agents.

- $\mathbf{S}:=\left(S_{1}, \ldots, S_{n}\right)$, where $S_{i}$ denotes the set of pure strategies that agent $i$ may play.

- For convenience, let $S:=\bigcup_{i} S_{i}=\left\{s_{1}, \ldots, s_{|S|}\right\}$ denote the set of all strategies, and thus each $S_{i} \subseteq S$. Also, we write $S_{i}=\left\{s_{i, 1}, s_{i, 2}, \ldots, s_{i,|S|_{i}}\right\}$ Furthermore, we'll let $s(i)$ denote the strategy played by agent $i$.

- For any $S^{\prime} \subset S$, let $\Delta\left(S^{\prime}\right)$ denote the set of valid configurations of agents to strategies $s \in S^{\prime}$; we represent a configuration $D\left(S^{\prime}\right) \in \Delta\left(S^{\prime}\right)$ as an $\left|S^{\prime}\right|$-tuple $D\left(S^{\prime}\right)=\left\{n_{1}, \ldots, n_{\left|S^{\prime}\right|}\right\}$ where $n_{i}$ is the number of agents playing the $i^{\text {th }}$ strategy of $S^{\prime}$.

- $G$ is a directed graph with one node for each action $s_{i}$. Let $\nu: S \rightarrow 2^{S}$, be the neighbor relation induced by graph $G$, where $s^{\prime} \in \nu(s)$ if the edge $\left(s^{\prime}, s\right) \in G$. Note that self-loops are allowed, and thus it is possible that $s \in \nu(s)$. We refer to $G$ as the strategy graph of $A$.

- The utility function $u$ assigns identical utilities to all agents playing a given strategy $s$, with the utility depending only on the number of agents playing neighboring strategies. Formally, $u: \Delta(S) \rightarrow \mathbb{R}^{|S|}$, via maps $u_{1}, \ldots, u_{|S|}$ where $u_{i}: \Delta\left[\nu\left(s_{i}\right)\right] \rightarrow \mathbb{R}$ defines the common utility of all agents playing strategy $s_{i}$.

Note that AGGs are fully expressive because any games can be written as an action graph game in which the strategy sets of different players are disjoint, and the strategy graph $G$ is complete.

We now define a further type of possible symmetry between agents that will be important in our analysis of the complexity of computing Nash equilibria.

Definition 2. We say that an $A G G$ has $k$ player types if there exists a partition of the agents into $k$ sets $P_{1}, \ldots, P_{k}$, such that if $p, p^{\prime} \in P_{i}$, then $S_{p}=S_{p^{\prime}}$. (The terminology of [15] refers to such games as $k$-symmetric AGGs.)

Since agents who play the same strategy receive the same utility, all agents of a given type are identical—for example an AGG with a single player type is a symmetric game. While the number of player types does not significantly alter the description size, decreasing the number of player types constrains the space of possible Nash equilibria; this is the motivation for considering AGGs with few player types as a possible class of tractable games.

A strategy profile, $M:=\left[m_{1}, \ldots, m_{n}\right]$, with $m_{i}=\left(p_{i, 1}, \ldots, p_{i,\left|S_{i}\right|}\right)$ assigns to each agent a probability distribution over the possible strategies that the agent may play, with $\operatorname{Pr}\left[s(i)=s_{i, k}\right]=p_{i, k}$ where $s_{k}$ is the $k^{\text {th }}$ element of $S_{i}$. Thus a given strategy profile induces an expected utility for each player $\mathbb{E}[u \mid M]=\sum_{D \in \Delta} u(D) \operatorname{Pr}(D)$, where the probability is with respect to the strategy profile $M$.

Definition 3. A strategy profile $M$ is a Nash-equilibrium if no player can increase her expected utility by changing her strategy $m_{i}$ given the strategy profiles $m_{-i}$ of the other agents. That is, for all strategy profiles $m_{i}^{\prime} . \mathbb{E}\left[u_{i} \mid m_{-i}, m_{i}\right] \geq$ $\mathbb{E}\left[u_{i} \mid m_{-i}, m_{i}^{\prime}\right]$.

Definition 4. A strategy $m \in M$ is an $\epsilon$-Nash-equilibrium if no player can increase her expected utility by more than $\epsilon$ by changing her strategy profile.

Note that there is the slightly stronger definition of an $\epsilon$-Nash equilibrium in which, for all agents $i$, the expected utility of playing every strategy $s$ in the support of $m_{i}$ is at most $\epsilon$ less than the expected utility of playing a different $s^{\prime} \in S_{i}$. We do not stress the distinction, as our PTAS finds such an $\epsilon$-Nash equilibrium, and our hardness results apply to the weaker definition given above.

\section{PTAS}

Action graph games have properties of both anonymous games and graphical games. As such, one might expect that classes of AGGs that resemble tractable classes of anonymous or graphical games could have efficiently computable equilibria. For anonymous games, the symmetry imposed by the limited number of types implies the existence of a highly symmetric mixed equilibrium which seems easier to find than asymmetric equilibria. For graphical games with small treewidth, the tree structure allows for an efficient message-passing dynamic-programming approach. For AGGs with a bounded number of player types and a strategy graph of constant treewidth, we give a PTAS for computing $\epsilon$-Nash equilibria that uses both the symmetry implied by bounding the number of player types, and a dynamic programming approach that exploits the tree structure. While these conditions might seem strong, we show in Section 3 that if either condition is omitted the problem of computing an $\epsilon$-Nash equilibrium is hard. 
Theorem 1. For any fixed constants $d, k$, and $t$, an $A G G A$ with $k$ player types and strategy graph $G_{A}$ with bounded degree $d$ and treewidth $t$, an $\epsilon$-Nash equilibrium can be computed in time polynomial in $|A|, 1 / \epsilon$.

We begin with a fact about games with few player types.

Fact 2 [18] Any AGG with $k$ player types has a Nash equilibrium where all players of a given type play identical mixed strategy profiles. Formally, there is a strategy profile $M=\left[m_{1}, \ldots, m_{n}\right]$ such that if $S_{i}=S_{j}$, then $m_{i}=m_{j}$. We refer to such equilibria as type-symmetric equilibria.

The the high-level outline of the PTAS is as follows: we discretize the space of mixed strategy profiles such that each player may play a given strategy with probability $N \delta$ for $N \in \mathbb{N}$, and some fixed $\delta>0$ that will depend on $\epsilon$ and $n$. We also discretize the space of target expected utilities into the set $V=\{0, \epsilon / 2, \epsilon, \ldots, 1\}$. Then, for each $i \in\{0, \ldots,|V|\}$, starting from the leaves of the strategy-graph tree, we employ dynamic programming to efficiently search the discretized strategy space for a type-symmetric $\epsilon$-Nash equilibrium in which each strategy in the support has an expected utility close to $v_{i}$. To accomplish this we associate to each strategy $s_{i}$ a polynomially sized table expressing the set of probabilities with which $s_{i}$ could be played so that some assignment of probabilities to the strategies below $s_{i}$ in the strategy tree could be extended to such an $\epsilon$-Nash equilibrium for the whole game. The following lemma guarantees the existence of such a type-symmetric $\epsilon$-Nash equilibrium.

Lemma 1. Given an n-player $A G G A$, with 1 player type and strategy graph $G_{A}$ with maximum degree $d$, for any $\delta>0$ there is a strategy profile $Q=\left(q_{1}, \ldots, q_{|S|}\right)$ with each $q_{i}$ a multiple of $\delta$ and the property that if all agents play profile $Q$, for any strategy $s$ in the support of $Q, \mathbb{E}\left[u_{s} \mid Q\right] \geq \mathbb{E}\left[u_{s^{\prime}} \mid Q\right]-2 \delta d n$ for all $s^{\prime} \in S$.

The following standard fact will be necessary in our proof:

Fact 3 For binomially distributed random variables $X=B(n, p), Y=B(n, p+\delta)$

$$
\max _{k}|\operatorname{Pr}(X=k)-\operatorname{Pr}(Y=k)| \leq n \delta .
$$

Proof of Lemma 1: From Fact 2, there exists a strategy profile $P=\left(p_{1}, \ldots, p_{|S|}\right)$ which is a Nash equilibrium of $A$. Consider a strategy profile $Q$ with the property that $q_{i}=0$ if $p_{i}=0$, and otherwise $\left|q_{i}-p_{i}\right| \leq \delta$. (Note that such a profile clearly exists.)strategy $s_{i}$ with $\nu\left(s_{i}\right)=d$, we now show that

$$
\left|\mathbb{E}\left[u_{s} \mid Q\right]-\mathbb{E}\left[u_{s} \mid P\right]\right| \leq \delta d n,
$$

from which our lemma follows.

For a single neighbor $s_{j}$ of $s_{i}$, from Fact $3\left|\operatorname{Pr}\left(D\left(s_{j}\right)=k \mid Q\right)-\operatorname{Pr}\left(D\left(s_{j}\right)=k \mid P\right)\right| \leq \delta n$, and thus if we were to replace $p_{j}$ by $q_{j}$ in profile $P$, this change would affect the expectation of playing $s_{i}$ by at most $\delta n$. Applying this reasoning to each of the $d$ neighbors completes our proof.

We now describe the PTAS; for clarity we describe the algorithm in the case that $k=1$, and $G_{A}$ is a tree with maximum degree 3 , although it extends easily to a constant number of player types and constant treewidth. The following definition simplifies our description of the algorithm.

Definition 5. We say that some set of strategy profiles is an $\epsilon$-partial equilibrium for a subset $S^{\prime} \subset S$ of strategies if, for all strategies $s_{i}$ played with nonzero probability, the expected utility of playing $s_{i}$ is at most $\epsilon$ less than the expected utility of playing some other $s^{\prime} \in S^{\prime}$.

Consider a fixed $\epsilon>0$, and an $n$-player AGG $A=\langle P, \mathbf{S}, G, u\rangle$ with $k$ types, with strategies $S=\left\{s_{1}, \ldots, s_{|S|}\right\}$ and strategy graph $G_{A}$ that is a tree with maximum degree of 3 . Arbitrarily choose some strategy with degree 1 as the root of $G_{A}$, and without loss of generality denote it by $s_{1}$. Given a strategy $s_{i}$, let $s_{R(i)}, s_{L(i)}$ denote the right and left children of $s_{i}$ in the strategy graph. If $s_{i}$ has only one child, let $s_{R(i)}$ denote this child and $s_{L(i)}=n u l l$. Fix $\delta=\frac{\epsilon}{2 d n}$. Set $V:=\left\{v_{0}, \ldots, v_{|V|}\right\}$ where $v_{i}:=\frac{i \epsilon}{2}$.

Let $f_{i}$ represent a table of size $\frac{1}{\delta^{4}}|V|=\operatorname{poly}\left(\frac{1}{\epsilon}, n,|S|\right)$ associated with strategy $s_{i}$, which can be thought of as a function $f_{i}: I_{\delta}^{4} \times V \rightarrow\{0,1\}$ where $I_{\delta}=\{0, \delta, 2 \delta, \ldots, 1\}$. The function $f_{i}\left(p, p_{R}, p_{L}, w, v_{i}\right)$ will indicate whether there is a type-symmetric $\frac{\epsilon}{4}$-partial equilibrium in the set of strategies below $s_{i}$ in $G_{A}$ with expected utility near $v_{i}$ and strategies $s_{i}, s_{R(i)}, s_{L(i)}$ played with probabilities $p, p_{R}, p_{L}$, respectively, where the probability of choosing strategy 
$s_{i}$, or one that lies below $s_{i}$ is $w$. In addition to $f_{i}$, we also construct another table $g_{i}: I_{\delta}^{4} \times V \rightarrow I_{\delta}^{2}$ that will facilitate the reconstruction of an $\epsilon$-Nash equilibrium after having computed all the tables $f_{i}$ and $g_{i}$. The $g_{i}$ will record the total weight used in each of the partial solutions which were combined. A given element of $g_{i}$ will never be used if the corresponding element of $f_{i}$ is 0 , and for simplicity we neglect to define $g_{i}$ for these entries. Starting from the leaves, we calculate the tables $f_{i}, g_{i}$ as follows:

- if $s_{i}$ is a leaf, $f_{i}\left(p, p_{R}, p_{L}, w, v_{i}\right)=1$ iff $p=w$ and $p_{R}=p_{L}=0$

- if $s_{i} \neq s_{1}$ has one child, $f_{i}\left(p, p_{R}, p_{L}, w, v\right)=1$ and $g_{i}\left(p, p_{R}, p_{L}, w, v\right)=(w-p, 0)$ iff $p_{L}=0$ and there exist $q_{R}, q_{L}, w^{\prime} \in I_{\delta}$ such that the following conditions hold:

- $f_{R(i)}\left(p_{R}, q_{R}, q_{L}, w^{\prime}, v\right)=1$

- if $p_{R}>0$ the expected utility of playing $s_{R(i)}$ is in $\left(v-\frac{\epsilon}{2}, v+\frac{\epsilon}{2}\right)$ given that $s_{i}, s_{R(R(i))}, s_{L(R(i))}$ are played with respective probabilities $p, q_{R}, q_{L}$.

- if $p_{R}=0$ the expected utility of playing $s_{R(i)}$ is at most $v+\frac{\epsilon}{2}$ given that $s_{i}, s_{R(R(i))}, s_{L(R(i))}$ are played with respective probabilities $p, q_{R}, q_{L}$.

- $w=w^{\prime}+p$

- if $s_{i} \neq s_{0}$ has two children, $f_{i}\left(p, p_{R}, p_{L}, w, v\right)=1$ and $g_{i}\left(p, p_{R}, p_{L}, w, v\right)=\left(w^{R}, w^{L}\right)$ if there exist $q_{R}^{R}, q_{L}^{R}, w^{R}, q_{R}^{L}$ $q_{L}^{L}, w^{L} \in I_{\delta}$ such that the following conditions hold:

- $f_{R(i)}\left(p_{R}, q_{R}^{R}, q_{L}^{R}, w^{R}, v\right)=1=f_{L(i)}\left(p_{L}, q_{R}^{L}, q_{L}^{L}, w^{L}, v\right)$

- if $P_{R}>0$ the expected utility of playing $s_{R(i)}$ is in $\left(v-\frac{\epsilon}{2}, v+\frac{\epsilon}{2}\right)$ given that $s_{i}, s_{R(R(i))}, s_{L(R(i))}$ are played with respective probabilities $p, q_{R}, q_{L}$. Analogously for the utility of playing $s_{L(i)}$ if $p_{L}>0$.

- if $p_{R}=0$ the expected utility of playing $s_{R(i)}$ is at most $v+\frac{\epsilon}{2}$ given that $s_{i}, s_{R(R(i))}, s_{L(R(i))}$ are played with respective probabilities $p, q_{R}^{R}, q_{L}^{R}$. Analogously for the utility of playing $s_{L(i)}$ if $p_{L}=0$.

- $w=w^{R}+w^{L}+p$

Note that there may be multiple choices of $q_{R}^{R}, q_{L}^{R}, w^{R}, q_{R}^{L}, q_{L}^{L}, w^{L}$ that satisfy the above conditions, in which case $g_{i}\left(p, p_{R}, p_{L}, w, v\right)=\left(w^{R}, w^{L}\right)$ can be assigned to an arbitrary choice of such $w^{R}, w^{L}$.

- set $f_{1}\left(p, p_{R}, p_{L}, w, v\right)=1$ iff $p_{L}=0, w=1$, and there exist $q_{R}, q_{L}, w^{\prime} \in I_{\delta}$ such that the following conditions hold:

- $f_{R(1)}\left(p_{R}, q_{R}, q_{L}, w^{\prime}, v\right)=1$

- if $p_{R}>0$ the expected utility of playing $s_{R(1)}$ is in $\left(v-\frac{\epsilon}{2}, v+\frac{\epsilon}{2}\right)$ given that $s_{1}, s_{R(R(1))}, s_{L(R(1))}$ are played with respective probabilities $p, q_{R}, q_{L}$.

- if $p_{R}=0$ the expected utility of playing $s_{R(1)}$ is at most $v+\frac{\epsilon}{2}$ given that $s_{1}, s_{R(R(1))}, s_{L(R(1))}$ are played with respective probabilities $p, q_{R}, q_{L}$.

- if $s_{1}>0$ the expected utility of playing $s_{1}$ is in $\left(v-\frac{\epsilon}{2}, v+\frac{\epsilon}{2}\right)$ given that $s_{R(1)}$ is played with probability $p_{R}$.

- if $s_{1}=0$ the expected utility of playing $s_{1}$ is at most $v+\frac{\epsilon}{2}$ given that $s_{R(1)}$ is played with probability $p_{R}$.

- $w=w^{\prime}+p$

The following lemma ensures that the tables $f_{i}$ behave as hoped, and we find at least one approximate Nash equilibria. The proof follows from the definition above and induction on the tree structure. For the sake of brevity we omit a formal proof.

Lemma 2. Given a strategy profile $\left(q_{1}, \ldots, q_{|S|}\right)$ that is a $\frac{\epsilon}{4}$-Nash equilibrium of the form guaranteed in Lemma 1 , then for all $i, f_{i}\left(q_{i}, q_{R(i)}, q_{L(i)}, w, v\right)=1$, where $v$ is chosen to be a multiple of $\epsilon / 2$ and to be within $\epsilon / 2$ of the expected utility of playing any strategy in the support, and $w$ is the sum of the weights on strategy $i$ and its descendants in the tree.

The following two lemmas demonstrate that the tables $f_{i}$ can be computed efficiently, and that given the tables, an $\epsilon$-Nash equilibrium can be efficiently computed.

Lemma 3. The tables $f_{0}, \ldots, f_{|S|}$ and $g_{0}, \ldots, g_{|S|}$ can be computed efficiently.

Proof. The size of each table is polynomially sized. $f_{i}$ and $g_{i}$ can be computed efficiently given the tables $f_{R(i)}, f_{L(i)}$.

Lemma 4. Given the tables $f_{0}, \ldots, f_{|S|}$, and $\epsilon$-Nash equilibrium can be found efficiently. 
Proof. Starting from the root of the tree $G_{A}$, we will populate a strategy profile $\left(p_{1}, \ldots, p_{|S|}\right)$ that will be a typesymmetric $\epsilon$-Nash equilibrium. Lemmas 2 and 1 guarantee that there will be some choice of $p, p_{R} \in I_{\delta}$, and $v \in V$ such that $f_{1}\left(p, p_{R}, 0,1, v\right)=1$. We set $p_{1}=p, p_{R(1)}=p_{R}$, and 'pass' $w=1-p_{1}$ and $v$ to strategy $s_{R(1)}$. For all other strategies $s_{i}$, with $i \geq 2$ such that $p_{i}$ has already been fixed but $p_{R(i)}, p_{L(i)}$ have not been fixed yet, the parent of $s_{i}$ will have passed a pair $w, v$. In the case that $s_{i}$ has one child, from our construction of $f_{i}$, it follows that there must be a choice of $q_{R} \in I_{\delta}$ such that $f_{i}\left(p_{i}, q_{R}, 0, w-p_{i}, v\right)=1$. We then set $p_{R(i))}=q_{R}$ and $p_{L(i)}=q_{L}$ and pass the pair $w^{\prime}=w-p_{i}, v$ to $s_{R(i)}$. In the case that $s_{i}$ has two children, from our construction of $f_{i}$, it follows that there must be a choice of $q_{R}, q_{L} \in I_{\delta}$ such that $f_{i}\left(p_{i}, q_{R}, q_{L}, w, v\right)=1$, and $g_{i}\left(p_{i}, q_{R}, q_{L}, w, v\right)=\left(w^{R}, w^{L}\right)$. We then set $p_{R(i))}=q_{R}, p_{L(i))}=q_{L}$, and pass the pairs $w^{R}, v$ and $w^{L}, v$ to the right and left children, respectively.

From our construction, it follows that $\sum_{i} p_{i}=1$, and that for every strategy $s_{i}$ in the support, the expected utility of playing that strategy is at most $\epsilon$ less than the expected utility of playing any other strategy. In particular, $\left(p_{1}, \ldots, p_{|S|}\right)$ is an $\epsilon$-Nash equilibrium.

This algorithm and proof easily extends to the case where there are a constant $k$ player types: simply create tables $f_{i}^{(j)}$ and $g_{i}^{(j)}$ for each type $j: 1 \leq j \leq k$ and strategy $i: 1 \leq j \leq|S|$ and proceed analogously but additionally require that $f_{i}^{j}\left(p_{i}, p_{R}, p_{L}, w^{j}, v^{j}\right)=0$ if $i \notin S_{j}$ and $p_{i} \neq 0$. That is, enforce that each player type only play the strategies available.

Additionally, using a standard technique, the algorithm extends to the case where the tree-width is bounded by some constant $t$. Intuitively, in this case the strategy graph decomposes into a tree over cliques of size $t$ of vertices on the graph. All the vertices in each clique are processed simultaneously. Because $t$ is constant, the increase in running time is polynomial.

Finally, one could consider the setting in which there is an unbounded number of player types, but each type consists of a connected region of the tree. An analogue of the above algorithm can handle this setting provided not too many player types can play any particular strategy.

Definition 6. Let $S_{1}, \ldots, S_{k}$ be subset of a vertices of a tree $T$. Define $S_{i}^{c}$ to be smallest connected region of $T$ such that $S_{i} \subseteq S_{i}^{c}$. We define the overlap of $S_{1}, \ldots, S_{k}$ of $T$ to be $\max _{t \in T}\left|i: t \in S_{i}^{c}\right|$.

Corollary 1. For any fixed constants $c$ and $d$, an $A G G A$ with $k$-player types $S_{1}, \ldots, S_{k}$, and strategy graph $G_{A}$ which is a tree with bounded degree 1 , and the overlap of $S_{1}, \ldots, S_{k}$ on $G_{A}$ is at most $c$, an $\epsilon$-Nash equilibrium can be computed in time polynomial in $|A|, 1 / \epsilon$.

Proof. For each strategy $i \in S$, define tables $f_{i}^{(j)}$ and $g_{i}^{(j)}$ for type $j$ only when $i \in S_{j}^{c}$ and proceed as in the case of $k$ player types.

\section{Hardness Results}

In this section we state and prove our four hardness results. We show that it is (1) NP-complete to decide the existence of pure-strategy Nash equilibria, and (2) PPAD complete to approximate general (mixed Nash) equilibria for the classes of action graph games that either (a) have action graphs of treewidth 1 or (b) are symmetric (all agents are of a single type). Our two hardness results for pure equilibria will come from reductions from the NP-complete problem CIRCUITSAT, and follow the approach of [23]. Our hardness results for approximating mixed Nash equilibria are via equilibria-preserving gadgets that let us reduce from the PPAD-complete problem of computing equilibria in the class of graphical games where the maximum degree is 3 and each player has only two possible strategies. We begin by showing that action graph games are in the class PPAD.

\section{Mapping Action Graph Games to Graphical Games}

We show the following result which reduces the problem of computing a Nash equilibrium of an action graph game to the problem of computing a Nash equilibrium of a graphical game. Since the latter is in PPAD [19], it follows that the former is in PPAD as well. 
Theorem 4. Any action-graph game A can be mapped in polynomial time to a graphical game $\mathcal{G}$ so that there is a polynomial-time computable surjective mapping from the set of Nash equilibria of $\mathcal{G}$ to the set of Nash equilibria of $A$.

Proof. Let us define a bounded division-free straight-line program to be an arithmetic binary circuit with nodes performing addition, subtraction, or multiplication on their inputs, or evaluating to pre-set constants, with the additional constraint that the values of all the nodes remain in $[0,1]$.

We will show that there exists a bounded division-free staight-line program of polynomial size in the description of the action graph game which, given a mixed strategy profile $M:=\left\{\left(p_{i, 1}, \ldots, p_{i,\left|S_{i}\right|}\right)\right\}_{i=1}^{n}$, computes, for every agent $i, i=1, \ldots, n$, and for every pure strategy $s_{i}, s_{i} \in S_{i}$, of that agent, the expected utility that this agent gets for playing pure strategy $s_{i}$. The proof then follows from Theorems 1 and 2 of [7].

Without loss of generality, we will show that there exists a straight-line program of polynomial size for computing the expected utility of agent 1 for playing pure strategy $s_{1,1}$. For this purpose, let $\mathcal{N}:=\nu\left(s_{1,1}\right)$ and $\Delta:=\Delta\left(\nu\left(s_{1,1}\right)\right)$. Also, for any subset $P^{\prime} \subseteq P$ of the agents, let $\Delta_{P^{\prime}}$ be the set of valid configurations of the agents of the set $P^{\prime}$ to the strategies in $\mathcal{N}$, represented as $|\mathcal{N}|$-tuples of numbers; then, for every $D \in \Delta_{P^{\prime}}$, let $\operatorname{Pr}_{P^{\prime}}[D]$ be the probability that configuration $D$ arises from the set of agents $P^{\prime}$, where the measure $\operatorname{Pr}_{P^{\prime}}$ is taken over the mixed strategies of agents in $P^{\prime}$. Using this notation, the expected payoff of agent 1 for playing $s_{1,1}$ can be written as follows

$$
\mathcal{U}_{1, s_{1,1}}:=\sum_{D \in \Delta_{P} \backslash\{1\}} u_{s_{1,1}}\left(D+1_{s_{1,1}}\right) \operatorname{Pr}_{P \backslash\{1\}}[D],
$$

where $1_{s_{1,1}}$ is an $|\mathcal{N}|$-tuple of numbers having a 1 at the coordinate corresponding to strategy $s_{1,1}$ and 0 everywhere else, and where $D+1_{s_{1,1}}$ represents coordinate-wise addition.

From Equation (1), it follows that, if there is a bounded division-free straight-line program of polynomial size which computes the values $\left\{\operatorname{Pr}_{P \backslash\{1\}}[D]\right\}_{D \in \Delta_{P \backslash\{1\}}}$, a straight-line program for computing $\mathcal{U}_{1, s_{1,1}}$ can be constructed at an additional cost of $O\left(\left|\Delta_{P \backslash\{1\}}\right|\right)=O(|A|)$ arithmetic gates. To conclude the proof, we prove the following lemma.

Lemma 5. Let $P_{j}=\{j+1, \ldots, n\} \subseteq P$ and suppose that there exists a bounded division-free staight-line program computing the values $\left\{\operatorname{Pr}_{P_{j}}[D]\right\}_{D \in \Delta_{P_{j}}}$. Also, suppose that the size of this straight-line program is bounded by $g$. Then there exists a straight-line program of size bounded by $g+O(|A| \cdot(|A|+|S|))$ which computes the values $\left\{\operatorname{Pr}_{P_{j} \cup\{j\}}[D]\right\}_{D \in \Delta_{P_{j} \cup\{j\}}}$.

Proof. Note first that $\left|\Delta_{P_{j} \cup\{j\}}\right|=O(|A|)$, where $|A|$ is the description size of the action graph game. Then, for every $D \in \Delta_{P_{j} \cup\{j\}}$, it holds that

$$
\begin{gathered}
\operatorname{Pr}_{P_{j} \cup\{j\}}[D]=\sum_{s_{j, k} \in S_{j} \backslash \mathcal{N}} \operatorname{Pr}_{\{j\}}\left[j \text { plays } s_{j, k}\right] \operatorname{Pr}_{P_{j}}[D] \\
+\sum_{\substack{s_{j, k} \in S_{j} \cap \mathcal{N}, D^{\prime} \in \Delta_{P_{j}}: \\
1_{s_{j, k}}+D^{\prime}=D}} \operatorname{Pr}_{\{j\}}\left[1_{s_{j, k}}\right] \operatorname{Pr}_{P_{j}}\left[D^{\prime}\right],
\end{gathered}
$$

where in the above expression $\operatorname{Pr}_{P_{j}}[D]=0$, if $D \notin \Delta_{P_{j}}$. Observe that in (2) the first summation has at most $|S|$ terms and the second at most $|A|$ terms. Hence, given the values $\left\{\operatorname{Pr}_{P_{j}}[D]\right\}_{D \in \Delta_{P_{j}}}$, the above expression can be evaluated with $O(|A|+|S|)$ arithmetic operations. The result follows.

From the lemma above it follows that there exists a straight-line program of size bounded by $O(n \cdot|A| \cdot(|A|+|S|))=$ $O\left(|A|^{3}\right)$ for computing $\left\{\operatorname{Pr}_{P \backslash\{1\}}[D]\right\}_{D \in \Delta_{P \backslash\{1\}}}$. This concludes the proof of the theorem, since all the intermediate values of the computations described above are in $[0,1]$.

\section{A Copy Gadget}

As a preliminary to the hardness results of the next two subsections, we describe a copy gadget which will prove useful in both NP-completeness and PPAD-completeness results. Intuitively, to simulate games $G$ of high treewidth by treewidth 1 action graph games $H$, we create several "copies" of each player, but only one copy of each edge 
relating players, thus ending up with a very "sparse" simulation, whose treewidth we can control. Explicitly, given an AGG $A$, and an agent $i$ whose strategy set consists of the two strategies $S_{i}=\left\{f_{i}, t_{i}\right\}$, our copy gadget will add two additional players $a, c$, of which player $c$ will be the "copy" and player $a$ is an auxiliary player, whose inclusion will allow player $i$ 's strategies to be disconnected from player $c$ 's. We add strategies for $a$ and $c$ that are $\left\{f_{a}, t_{a}\right\}$ and $\left\{f_{c}, t_{c}\right\}$ respectively, and set the incentives so that in any Nash equilibrium $\operatorname{Pr}\left[s(i)=t_{i}\right]=\operatorname{Pr}\left[s(b)=t_{b}\right]$ (and $\left.\operatorname{Pr}\left[s(i)=f_{i}\right]=\operatorname{Pr}\left[s(b)=f_{b}\right]\right)$.

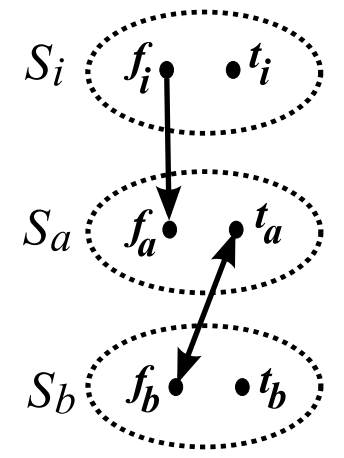

Fig. 1. The copy gadget-in any Nash equilibrium $\operatorname{Pr}\left[s(b)=f_{b}\right]=\operatorname{Pr}\left[s(i)=f_{i}\right]$.

Definition 7. Given an $A G G A=\langle P, \mathbf{S}, G, u\rangle$, and an agent $i$ with two strategy choices $S_{i}=\left\{f_{i}, t_{i}\right\}$, we create $A G G A^{\prime}=\left\langle P^{\prime}, \mathbf{S}^{\prime}, G^{\prime}, u^{\prime}\right\rangle$ from $A$ via the addition of a copy gadget on $i$ as follows:

- $P^{\prime}:=P \cup\{a, c\}$.

- $\mathbf{S}^{\prime}:=\left(S_{1}, \ldots, S_{|P|}, S_{a}, S_{c}\right)$, where $S_{a}=\left\{f_{a}, t_{a}\right\}$, and $S_{c}=\left\{f_{c}, t_{c}\right\}$, where $f_{a}, t_{a}, f_{c}, t_{c} \notin S$.

- $G^{\prime}$ consists of the graph $G$ with the additional vertices corresponding to $f_{a}, t_{a}, f_{c}, t_{c}$, and the directed edges $\left(f_{i}, f_{a}\right),\left(t_{a}, f_{c}\right),\left(f_{c}, t_{a}\right)$.

- $u^{\prime}$ is identical to u for all strategies in $S^{\prime} \backslash\left\{S_{a} \cup S_{c}\right\}$, and for a configuration $D, u^{\prime}\left(f_{a}\right)=D\left(f_{i}\right), u^{\prime}\left(t_{a}\right)=$ $1-D\left(f_{c}\right)$, and $u^{\prime}\left(f_{c}\right)=1-2 D\left(t_{a}\right)$.

See Figure 1 for a depiction of the copy gadget.

Lemma 6. Given an $A G G A$ and an agent $i$, the addition of a copy gadget on $i$ yields $A^{\prime}$ that satisfies the following properties:

- The description size of $A^{\prime}$ is at most a constant larger than $A$.

- In the strategy graph $G_{A^{\prime}}, f_{c}$ and $t_{c}$ are not path connected to either $f_{i}$ or $t_{i}$.

- In every $\epsilon^{2}$-Nash equilibrium with agent $i$ 's profile $\left(p_{i, f}, 1-p_{i, f}\right)$, agent c's profile will have $\left|p_{c, f}-p_{i, f}\right| \leq \epsilon$ (and $\left|p_{b, t}-p_{i, t}\right| \leq \epsilon$ ).

Proof. The first two properties follow directly from Definition 7. For the third property, assume otherwise and consider the case where $p_{c, f}>\epsilon+p_{i, f}$. Agent $a$ 's expected utility for playing $f_{a}$ is $p_{i, f}$, and is $p_{c, f}$ for playing $t_{a}$, thus our assumption that $p_{c, f}>\epsilon+p_{i, f}$ implies that agent $a$ must be playing $t_{a}$ with probability at least $1-\epsilon$ since the game is at $\epsilon^{2}$-equilibrium. Given that $a$ plays $t_{a}$ with probability at least $1-\epsilon$, agent $b$ maximizes her utility by playing $t_{c}$, and thus $p_{c, f} \leq \epsilon$, which contradicts our assumption that $p_{c, f}$ is the larger of $p_{c, f}, p_{i, f}$, namely at least $\frac{1}{2}$. An analogous argument applies to rule out the case $p_{c, f}<p_{i, f}-\epsilon$.

\subsection{PPAD-Completeness}

Our PPAD-Completeness results are reductions from the problem of computing equilibria in graphical games, and rely on the following fact due to [4]. 
Fact 5 For the class of graphical games with $n$ players, maximum degree 3 and payoffs in $\{0,1,2\}$, it is PPADcomplete to compute $\epsilon$-Nash equilibria where $\epsilon \propto 1 / \operatorname{pol} y(n)$.

Theorem 6. Computing a Nash equilibrium for AGGs with strategy graph $G_{A}$ is PPAD-complete even if treewidth $\left(G_{A}\right)=1$, and $G_{A}$ has constant degree.

Proof. From Theorem 4 this problem is in PPAD.

To show PPAD-hardness, we reduce from the known PPAD-hard problem of Fact 5. Given an instance of such a graphical game $H$, we construct an AGG $A_{H}^{\prime}$ with treewidth 1 and maximum degree 4 with similar description size to $H$ such that there a polynomial time mapping from $\epsilon$-Nash equilibria of $A_{H}^{\prime}$ to the $\epsilon$-Nash equilibria of $H$. We construct $A_{H}^{\prime}$ via the intermediate step of constructing an AGG $A_{H}$ which will be equivalent to $H$ and might have large treewidth. From $A_{H}$, we construct $A_{H}^{\prime}$ using our copy gadget to reduce the treewidth of the associated strategy graph. See Figure 2 for a depiction of the reduction.

The construction of $A_{H}$ is straightforward: for each player $i_{H}$ in the graphical game, we have a corresponding player $i_{A}$ in the AGG with strategy set $S_{i_{A}}=\left\{f_{i}, t_{i}\right\}$, corresponding to the two strategies that $i_{H}$ may play in $H$. For each undirected edge between players $(i, j) \in H$, we add directed edges between the $t$ nodes $\left(t_{j}, t_{i}\right),\left(t_{i}, t_{j}\right)$, and edges between the $f$ nodes $\left(f_{j}, f_{i}\right),\left(f_{i}, f_{j}\right)$ to the strategy graph $G_{A_{H}}$ of $A_{H}$. We define utilities $u$ by simulating the utility functions from the original game $H$ : from each $f$ strategy connected to $i_{A}$ in the AGG we know that if it is played then the corresponding $t$ strategy is not played and vice versa; thus we have recovered the strategy choice of each neighbor of $I_{H}$ in original graphical game; we then apply the utility function of the graphical game to compute the utility in the AGG. We do the symmetric procedure for the $t$ nodes of the AGG. From the construction, it is clear that $H$ and $A_{H}$ represent the same game via the correspondence $i_{H} \rightarrow i_{A}$, and in particular an $\epsilon$-Nash equilibrium of one game will correspond to an $\epsilon$-Nash equilibrium of the other game via the natural mapping.

We obtain $A_{H}^{\prime}$ from $A_{H}$ by making three copies of each $i_{A}$ via the copy gadget. Thus for each $i$ there are agents $i_{A}, i_{A}^{1}, i_{A}^{2}, i_{A}^{3}$ with $S_{i_{A}^{k}}=\left\{f_{i_{A}}^{k}, t_{i_{A}}^{k}\right\}$. Finally, for each of the (at most three) outgoing edges of $f_{i_{A}}$ that are not part of copy gadgets, i.e the edges of the form $\left(f_{i_{A}}, f_{j_{A}}\right)$, we replace the edge by $\left(f_{i_{A}}^{k}, f_{j_{A}}\right)$, with each $f_{i_{A}}^{k}$ having at most one outgoing edge, and modify the utility function $u$ analogously so as to have the utility of strategy $f_{j_{A}}$ depend on $f_{i_{A}}^{k}$ instead of $f_{i_{A}}$. Analogous replacements are made for the outgoing edges of $t_{i_{A}}$. Since the copied strategies $f_{i_{A}}^{k}, t_{i_{A}}^{k}$ are disconnected from the original strategies $f_{i_{A}}, t_{i_{A}}$ the longest path in the strategy graph $G_{A_{H}^{\prime}}$ associated with $A_{H}^{\prime}$ has length at most 4, with maximum degree 6 , and $\operatorname{treewidth}\left(G_{A_{H}}\right)=1$. (See Figure 2.) Lemma 6 guarantees that the transformation from $A_{H}$ to $A_{H}^{\prime}$ increases the representation size by at most a constant factor. Further, from an $\frac{1}{144} \epsilon^{2}$-Nash equilibrium of $A_{H}^{\prime}$ we can extract an $\epsilon$-Nash equilibrium of $A_{H}$ by simply ignoring the new players: all of the copies $i_{A}^{k}$ of a player $i_{A}$ will play strategies with probabilities within $\frac{1}{12} \epsilon$ of the probabilities of playing the original by Lemma 6; thus the joint distribution of any triple of these will have joint distribution within $\frac{1}{4} \epsilon$ of the "true" joint distribution; since each utility has magnitude at most 2 the computed utilities will be within $\frac{1}{2} \epsilon$ of the utilities computed in $A_{H}$; thus each of the mixed strategies of a player $i_{A}$ in $A_{H}^{\prime}$, interpreted as a strategy in $A_{H}$ will yield utility within $\epsilon$ of optimal. From Fact 5 we conclude that finding an $\frac{1}{144} \epsilon^{2}-$ Nash equilibrium of $A_{H}^{\prime}$ is PPAD complete for any polynomial $\epsilon$, yielding the desired result.

We now turn our attention to AGGs that have a constrained number of player types.

Theorem 7. Computing a Nash equilibrium for symmetric AGGs (1 player type) is PPAD-complete even if the strategy graph $G_{A}$ has bounded degree.

To show PPAD-hardness, as above we reduce from the known PPAD-hard problem of computing Nash equilibria in graphical games of degree at most 3 where each player chooses between 2 strategies $f, t$ and has utility 0,1 , or 2 . Given such a graphical game $H$, we will reduce it to an AGG $A_{H}$ that has strategies $f_{i}, t_{i}$ corresponding to the two strategies that agent $i$ may choose in $H$. Intuitively, if our reduction is to be successful there are several properties of $G_{H}$ that seem necessary. First, in every Nash equilibrium of $G_{H}$, there must be at least one agent playing either $f_{i}$ or $t_{i}$ for every $i$. This is accomplished by giving agents a bonus payment if they choose either of the two strategies of a sparsely-played $f_{i}, t_{i}$ pair. Second, there must be some unambiguous mapping between the number of agents playing $f_{i}$ and $t_{i}$ in $A_{H}$ to a choice of actions of agent $i$ in $H$. This is accomplished via the MAJORITY function: if more agents play $f_{i}$ than $t_{i}$ in $A_{H}$, we say that $i$ plays $f$. This motivating intuition is formalized in the proof below.

Proof of Theorem 7: From Theorem 4 this problem is in PPAD

To show PPAD-hardness, we reduce from the known PPAD-hard problem of computing Nash equilibria in graphical games of degree at most 3 where each player chooses between 2 strategies and has utility 0,1 , or 2 . Given an 


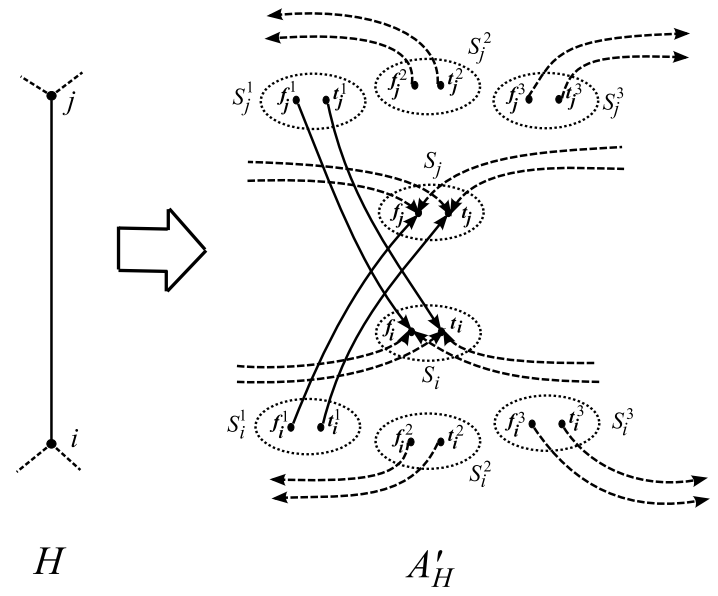

Fig. 2. The transformation from the graphical game $H$ to the AGG $A_{H}^{\prime}$. For simplicity, the internal strategies and edges associated with the copy gadgets are omitted.

instance of such a graphical game $H$, with $n$ agents, and some $\epsilon>0$ we construct the symmetric AGG $A_{H}$ so that an $\epsilon-$ Nash equilibrium of $A_{H}$ can be efficiently mapped to a $2 \epsilon-$ Nash equilibrium of $H$. We construct $A_{H}=\left\langle P, \mathbf{S}, G_{A}, u\right\rangle$ as follows:

- $P:=\{1, \ldots, 3 c n\}$ with $c>\frac{64}{\epsilon^{2}}$.

- $\mathbf{S}:=(S, \ldots, S)$, that is, each player has identical (symmetric) strategy set $S:=\left\{f_{1}, t_{1}, \ldots, f_{n}, t_{n}\right\}$ where strategies $f_{i}$ and $t_{i}$ correspond to the two strategies of the $i^{\text {th }}$ agent of $H$.

- For every undirected edge $(i, j)$ in the graph of $H$, the strategy graph $G_{A}$ has the eight directed edges $\left(f_{i}, f_{j}\right)$, $\left(f_{j}, f_{i}\right),\left(f_{i}, t_{j}\right),\left(t_{j}, f_{i}\right),\left(t_{i}, f_{j}\right),\left(f_{j}, t_{i}\right),\left(t_{i}, t_{j}\right),\left(t_{j}, t_{i}\right)$. Furthermore, for all $i \in\{1, \ldots, n\}, G_{A}$ contains the edges $\left(f_{i}, t_{i}\right),\left(t_{i}, f_{i}\right)$ and the self loops $\left(f_{i}, f_{i}\right)$ and $\left(t_{i}, t_{i}\right)$.

- To simplify the description of the utility function $u$, it will be useful to define the indicator functions $I_{1}\left[D\left(f_{1}, t_{1}\right)\right], \ldots, I_{n}\left[D\left(f_{n}, t_{n}\right)\right]$ where

$$
I_{i}\left[D\left(f_{i}, t_{i}\right)\right]:=\left\{\begin{array}{l}
f \text { if } D\left(f_{i}\right) \geq D\left(t_{i}\right) \\
t \text { if } D\left(f_{i}\right)<D\left(t_{i}\right)
\end{array}\right.
$$

Let $u$ assign utility to $f_{i}$ as a function of $D(\nu(i))$, where $\nu(i)$ denotes $i$ 's neighbors, by applying the utility function for agent $i$ from $H$ on the simulated actions of her neighbors $j_{1}, j_{2}, j_{3}$ evaluated as $I_{j_{1}}\left[D\left(f_{j_{1}}, t_{j_{1}}\right)\right], I_{j_{2}}\left[D\left(f_{j_{2}}, t_{j_{2}}\right)\right]$, and $I_{j_{3}}\left[D\left(f_{j_{3}}, t_{j_{3}}\right)\right]$, respectively. Finally, if $D\left(f_{i}\right)+D\left(t_{i}\right) \leq c, u$ assigns an extra 100 utility to strategies $f_{i}$ and $t_{i}$.

Observe that the description size of $A_{H}$ is polynomial in $c n$, and thus is polynomial in the description size of $H$. From Fact 5 our theorem will follow if we show that any $\epsilon^{2}-$ Nash equilibrium of $A_{H}$ can be efficiently mapped to an $\epsilon-$ Nash equilibrium of $H$.

Consider the map from mixed strategy profiles of $A_{H}$ to mixed strategy profiles of $H$ given by $\phi: \mathbf{M}_{\mathbf{A}} \rightarrow \mathbf{M}_{\mathbf{H}}$ that assigns $M_{H}=\left[\left(p_{1, f}, 1-p_{1, f}\right), \ldots,\left(p_{n, f}, 1-p_{n, f}\right)\right]$ by setting $p_{i, f}:=\operatorname{Pr}_{M_{A}}\left(I_{i}=f\right)$ where the probability is taken over the distribution over $\Delta$ defined by $M_{A}$. It is clear that the map $\phi$ can be computed efficiently, as it essentially involves simply evaluating multinomial distributions on 4 outcomes.

Before showing that $\phi$ maps $\epsilon$-equilibria to $2 \epsilon$-equilibria we first show that the "extra utility" of 100 correctly incentivizes a large number of players to play on each strategy pair. We observe that in any mixed strategy profile there will be at least one agent, $j$, who has probability at most $1 / 3$ of receiving a payoff of at least 100 . Since his payoff from the simulation of $H$ is at most 2, such an agent's expected utility is at most $35+\frac{1}{3}$, and thus any Nash equilibrium mixed strategy profile must satisfy $100 \operatorname{Pr}\left(D\left(f_{i}\right)+D\left(t_{i}\right)<c\right)<36, \forall i \in\{1, \ldots, n\}$. If this were not the case, then agent $j$ could improve her expected utility to at least 36 by always choosing strategy $f_{i}$, a contradiction. The above inequality implies that

$$
\mathbb{E}\left[\max \left(D\left(f_{i}\right), D\left(t_{i}\right)\right)\right]>\frac{c}{4} .
$$


We now proceed with the proof of correctness of the map $\phi$. Let $M_{A}$ be an $\epsilon$-Nash equilibrium of $A_{H}$, and $M_{H}=\phi\left(M_{A}\right)$. Consider a player $i$ in the graphical game, and a strategy of his that he plays with probability at least $\epsilon$. Without loss of generality let this strategy be $f_{i}$. We show that his utility for playing $f_{i}$ is at least his utility for playing his other choice, $t_{i}$, minus $\epsilon$; taken together, these statements imply that $M_{H}$ is an $\epsilon$-Nash equilibrium of the graphical game, as desired.

Since $\mathbb{E}\left[\max \left(D\left(f_{i}\right), D\left(t_{i}\right)\right)\right]>\frac{c}{4}$, we have that if $f_{i}$ is played with probability at least $\epsilon$, namely if $\operatorname{Pr}\left(D\left(f_{i}\right) \geq\right.$ $\left.D\left(t_{i}\right)\right) \geq \epsilon$ then (by Chernoff bounds) we must have $\mathbb{E}\left[D\left(f_{i}\right)\right] \geq \frac{c}{6}$. This implies that for at least one of the $3 \mathrm{cn}$ players $j$ in $A_{H}$, his probability of playing $f_{i}$ is at least $\frac{1}{18 n}$, which is at least $2 \epsilon$. Thus, since $M_{A}$ is, by assumption, an $\epsilon^{2}$-Nash equilibrium, we have that player $j$ 's utility for playing $f_{i}$ is at most $\frac{1}{2} \epsilon$ below his utility for playing $t_{i}$, when the other players play from $M_{A}$. Further, by construction, each of these two utilities are within $\frac{1}{4} \epsilon$ of the utilities for player $i$ in the graphical game to play $f_{i}, t_{i}$ respectively, when the other players play from $M_{H}$ : this is because fixing player $j$ 's move from $M_{A, j}$ to one of $f_{i}, t_{i}$ changes each $I_{k}$ by at most the probability of $j$ playing one of $\left(f_{k}, t_{k}\right)$ divided by the standard deviation of $\max \left(D\left(f_{i}\right), D\left(t_{i}\right)\right)$, which is at least the square root of its expectation, $\sqrt{\frac{c}{4}}=\frac{4}{\epsilon}$. Thus by the triangle inequality we have that player $i$ 's utility for playing $f_{i}$ is no more than $\epsilon$ worse than playing $t_{i}$. Since our choice of $i$ was arbitrary and a corresponding argument applies to the $t$ strategies, we conclude that $M_{H}$ is an $\epsilon-$ Nash equilibrium of $H$, as desired.

\subsection{NP-Completeness}

Both of our NP-completeness results are reductions from the NP-Complete problem CIRCUITSAT and follow an approach employed in [23].

Fact 8 It is NP-complete to decide satisfiability for the class of circuits consisting of AND, OR, and NOT gates, with maximum degree 3 (in-degree plus out-degree).

In our reductions from CIRCUITSAT, given a circuit $C$, we construct an AGG $A_{C}$ that computes $C$ in the sense that pure strategy Nash equilibria of $A_{C}$ map to valid circuit evaluations. To this game we add two agents that have a simple pure-strategy equilibrium if $C$ evaluates to true, but when $C$ evaluates to false play pennies-a simple game that has no pure strategy Nash equilibria. Thus the existence of a pure strategy Nash equilibrium is equivalent to the satisfiability of $C$.

Theorem 9. Deciding the existence of a pure strategy Nash equilibrium for AGGs with strategy graph $G_{A}$ is $N P$ complete even if treewidth $\left(G_{A}\right)=1$, and $G_{A}$ has constant degree.

Proof. Membership in NP is clear. To show hardness, given a circuit $C$, we construct the associated AGG $A_{C}:=$ $\left\langle P, \mathbf{S}, G_{A}, u\right\rangle$ as follows:

- $P:=\left\{1, \ldots, n, p_{1}, p_{2}\right\}$, where $n$ is the number of gates in $C$, and the gate corresponding to player $n$ is the output gate.

- $\mathbf{S}:=\left(\left(f_{1}, t_{1}\right), \ldots,\left(f_{n}, t_{n}\right),\left(f_{p_{1}}, t_{p_{1}}\right),\left(f_{p_{2}}, t_{p_{2}}\right)\right)$.

- For every pair of gates $i, j$ for which the output of gate $i$ is an input to gate $j, G_{A}$ has the edges $\left(f_{i}, f_{j}\right)$, and $\left(f_{i}, t_{j}\right)$. Furthermore, we add edges $\left(f_{n}, f_{p_{1}}\right),\left(f_{n}, t_{p_{1}}\right),\left(f_{n}, f_{p_{2}}\right),\left(f_{n}, t_{p_{2}}\right)$, and the edges $\left(f_{p_{1}}, f_{p_{2}}\right),\left(f_{p_{1}}, t_{p_{2}}\right)$, $\left(f_{p_{2}}, f_{p_{1}}\right),\left(f_{p_{2}}, t_{p_{1}}\right)$.

- The utility function $u$ is defined as follows: if agent $i$ corresponds to an input gate, than strategies $f_{i}, t_{i}$ both have utility 0 . For any other agent $i$ corresponding to a gate of $C$, the payoff of strategy $f_{i}$ is 1 or 0 according to whether $f_{i}$ is the correct output value of gate $i$ given the values corresponding to the strategies played by neighboring agents/strategies. Similarly for the payoff for strategy $t_{i}$. If $D\left(f_{n}\right)=0$, then $f_{p_{1}}$ and $t_{p_{1}}$ have utility 0 , otherwise the utility of $p_{1}$ is 1 if $D\left(f_{p_{1}}\right)=D\left(f_{p_{2}}\right)$, and is 0 otherwise. The utility of $p_{2}$ is 1 if $D\left(f_{p_{1}}\right) \neq D\left(f_{p_{2}}\right)$, and is 0 otherwise.

From the construction it is clear that if $C$ is satisfiable, there is a pure strategy profile for agents $1, \ldots, n$ with agent $n$ playing $t_{n}$, such that agents $1, \ldots, n$ can not improve their utility by deviating from their strategies. Furthermore, $p_{1}$ will be indifferent between her strategies, and $p_{2}$ will play the opposite of $p_{1}$; in particular, there will be a pure strategy Nash equilibrium. If $C$ is not satisfiable, then any pure strategy profile that is an equilibrium for agents $1, \ldots, n$ will have $D\left(f_{n}\right)=1$, and thus $p_{1}$ will be incentivized to agree with $p_{2}$, and $p_{2}$ will be incentivized to disagree, and thus $A_{C}$ will admit no pure strategy Nash equilibrium. 
To complete the proof, note that we can apply the copy gadget to each agent of $A_{C}$, as was done in the proof of Theorem 6 to yield the game $A_{C}^{\prime}$ that has strategy graph of treewidth 1, and a mapping from equilibria of $A_{C}^{\prime}$ to equilibria of $A_{C}$.

Theorem 10. Deciding the existence of a pure strategy Nash equilibrium for symmetric AGGs (1 player type) is $N P$-complete even if the strategy graph $G_{A}$ has bounded degree.

Proof. Membership in NP is clear; to show hardness we proceed as was done in the proof of Theorem 9, and obtain AGG $A_{C}$ from circuit $C$. Now, we make $A_{C}$ symmetric by retaining the same number of agents, but allowing each of them to pick any of the strategies. We modify the strategy graph $G$ by adding edges $\left(f_{x}, t_{x}\right),\left(t_{x}, f_{x}\right),\left(f_{x}, f_{x}\right),\left(t_{x}, t_{x}\right)$ for each player $x$ from $A_{C}$, and extend the utility function $u$ so that if $D\left(f_{x}\right)+D\left(t_{x}\right)>1$ then strategies $f_{x}$ and $t_{x}$ have utility -1 . Thus in any pure strategy Nash equilibrium $D\left(f_{x}\right)+D\left(t_{x}\right)=1$, and the reasoning in the proof of Theorem 9 applies to complete our reduction.

\section{Conclusions and Open Problems}

We have presented results that further the understanding of the computational complexity of computing approximate Nash equilibria in action graph games. We provided a polynomial-time algorithm for finding approximate Nash equilibrium in action graph games under the restrictions that 1) the strategy graph of the game has constant degree and constant treewidth and 2) the players can by classified by a constant number of types. We showed that restricting the tree-width and the number of types of players is necessary to avoid the problem becoming PPAD-hard. Whether or not the restriction on the degree of the graph is necessary remains an open problem for future study.

We further showed the our algorithm extends to the case where the underlying graph is a bounded-degree tree and there are an arbitrary number of player types, but 1) each player type is a connected region of the graph and 2) each strategy is available to only a constant number of types. It remains an open problem if the second restriction is required. Furthermore, perhaps there are other restricted classes of games that circumvent the hardness result while retaining some of the motivating features of general action graph games.

We also study the complexity of computing pure Nash equilibria in action graph games with $k$-types. While Jiang and Brown in [15] show the problem is tractable provided 1) $k$ is constant and 2) the graph has bounded tree width, we show that both of these restrictions are necessary. Without either of them, finding pure Nash equilibrium in action graph games becomes $N P$-complete.

\section{References}

1. Navin A. R. Bhat and Kevin Leyton-Brown. Computing nash equilibria of action-graph games. In UAI, pages 35-42, 2004.

2. Matthias Blonski. Anonymous games with binary actions. Games and Economic Behavior, 28(2):171-180, August 1999. available at http://ideas.repec.org/a/eee/gamebe/v28y1999i2p171-180.html.

3. Xi Chen and Xiaotie Deng. Settling the complexity of two-player nash equilibrium. In FOCS '06: Proceedings of the 47th Annual IEEE Symposium on Foundations of Computer Science (FOCS'06), pages 261-272, Washington, DC, USA, 2006. IEEE Computer Society.

4. Xi Chen, Xiaotie Deng, and Shang-Hua Teng. Computing nash equilibria: Approximation and smoothed complexity. In FOCS, pages 603-612, 2006.

5. Steve Chien and Alistair Sinclair. Convergence to approximate nash equilibria in congestion games. In SODA, pages 169-178, 2007.

6. Bruno Codenotti and Daniel Štefankovič. On the computational complexity of nash equilibria for $(0,1)$ bimatrix games. Inf. Process. Lett., 94(3):145-150, 2005.

7. Constantinos Daskalakis, Alex Fabrikant, and Christos H. Papadimitriou. The game world is flat: The complexity of nash equilibria in succinct games. In ICALP (1), pages 513-524, 2006.

8. Constantinos Daskalakis, Paul W. Goldberg, and Christos H. Papadimitriou. The complexity of computing a nash equilibrium. In STOC, 2006.

9. Constantinos Daskalakis and Christos H. Papadimitriou. Computing pure nash equilibria in graphical games via markov random fields. In ACM Conference on Electronic Commerce, pages 91-99, 2006.

10. Constantinos Daskalakis and Christos H. Papadimitriou. Computing equilibria in anonymous games. In FOCS, 2007.

11. Constantinos Daskalakis and Christos H. Papadimitriou. On the exhaustive method for nash equilibria. Manuscript, 2007.

12. F. Fischer F. Brandt and M. Holzer. Equilibria of graphical games with symmetries. Technical Report TR07-136, Electronic Colloquium on Computational Complexity (ECCC), December 2007. 
13. Alex Fabrikant, Christos H. Papadimitriou, and Kunal Talwar. The complexity of pure nash equilibria. In STOC, pages 604-612, 2004.

14. D Gale, HW Kuhn, and AW Tucker. On symmetric games. Contributions to the Theory Games, Annals of Mathematics Studies, 24, 1950.

15. Albert Xin Jiang and Kevin Leyton-Brown. Computing pure nash equilibria in symmetric action graph games. In $A A A I$, pages 79-85. AAAI Press, 2007.

16. Ravi Kannan and Thorsten Theobald. Games of fixed rank: a hierarchy of bimatrix games. In SODA, pages 1124-1132, 2007.

17. C. E. Lemke and Jr J. T. Howson. Equilibrium points of bimatrix games. SIAM Journal of Applied Mathematics, 12:413-423, 1964.

18. John Nash. Non-cooperative games. Annals of Mathematics, 54(2):286-295, 1951.

19. Christos H. Papadimitriou. On the complexity of the parity argument and other inefficient proofs of existence. J. Comput. Syst. Sci., 48(3):498-532, 1994.

20. Christos H. Papadimitriou and Shmuel Safra. The complexity of low-distortion embeddings between point sets. In SODA, pages 112-118, 2005.

21. J. Rosenmuller. On a generalization of the lemke-howson algorithm to noncooperative n-person games. SIAM Journal of Applied Mathematics, 21:73-79, 1971.

22. H. E. Scarf. The approximation of fixed points of a continuous mapping. SIAM Journal of Applied Mathematics, 15:1328-1343, 1967.

23. Grant Schoenebeck and Salil Vadhan. The computational complexity of nash equilibria in concisely represented games. In $E C$ '06: Proceedings of the 7th ACM conference on Electronic commerce, pages 270-279, New York, NY, USA, 2006. ACM.

24. G. van der Laan and A. J. J. Talman. On the computation of fixed points in the product space of unit simplices and an application to noncooperative n person games. Mathematics of Operations Research, 7, 1982.

25. J. von Neumann and O. Morgenstern. On the computation of fixed points in the product space of unit simplices and an application to noncooperative $\mathrm{n}$ person games. Theory of Games and Economic Behavior, 1944.

26. R. Wilson. Computing equilibria of n-person games. SIAM Journal of Applied Mathematics, 21:80-87, 1971. 